\title{
A Novel Peptide Restricts Ethanol Modulation of the BK Channel In Vitro and In Vivo $\$$
}

\author{
Luisa L. Scott, Sangeetha lyer, Ashley E. Philpo, Melva N. Avalos, Natalie S. Wu, \\ Ted Shi, Brooke A. Prakash, Thanh-Tu Nguyen, S. John Mihic, Richard W. Aldrich, \\ and Jonathan T. Pierce
}

Waggoner Center for Alcohol and Addiction Research (L.L.S., S.I., A.E.P., M.N.A., N.S.W., T.S., B.A.P., T.-T.N., S.J.M., R.W.A., J.T.P.), Department of Neuroscience (S.J.M., R.W.A., J.T.P.), and Center for Learning and Memory (R.W.A., J.T.P.),

The University of Texas at Austin, Austin, Texas

Received July 6, 2018; accepted August 20, 2018

\begin{abstract}
Alcohol is a widely used and abused substance. A major unresolved issue in the alcohol research field is determining which of the many alcohol target proteins identified to date is responsible for shaping each specific alcohol-related behavior. The large-conductance, calcium- and voltage-activated potassium channel (BK channel) is a conserved target of ethanol. Genetic manipulation of the highly conserved $\mathrm{BK} \alpha$ channel influences alcohol-related behaviors across phylogenetically diverse species that include worm, fly, mouse, and man. A pharmacological tool that prevents alcohol's action at a single target, like the BK channel, would complement genetic approaches in the quest to define the behavioral consequences of alcohol at each target. To identify agents that specifically modulate the action of ethanol at the BK channel, we executed a high-throughput phagemid-display screen in combination with a
\end{abstract}

Caenorhabditis elegans behavioral genetics assay. This screen selected a novel nonapeptide, LS10, which moderated acute ethanol intoxication in a BK channel-humanized C. elegans strain without altering basal behavior. LS10's action in vivo was dependent upon BK channel functional activity. Singlechannel electrophysiological recordings in vitro showed that preincubation with a submicromolar concentration of LS10 restricted ethanol-induced changes in human $\mathrm{BK} \alpha$ channel gating. In contrast, no substantial changes in basal human BK $\alpha$ channel function were observed after LS10 application. The results obtained with the LS10 peptide provide proof-of-concept evidence that a combined phagemid-display/behavioral genetics screening approach can provide novel tools for understanding the action of alcohol at the BK channel and how this, in turn, exerts influence over central nervous system function.

\section{Introduction}

The large-conductance, calcium- and voltage-activated potassium channel (BK channel) is widely expressed in excitable cells, where it regulates muscle tone and neuronal signaling (Hoshi et al., 2013). The BK channel is a well-conserved target of ethanol in species as diverse as worm, fly, mouse, and man (Mulholland et al., 2009; Treistman and Martin, 2009; Bettinger and Davies, 2014). Pharmacologically relevant concentrations (10-100 $\mathrm{mM}$ ) of ethanol are sufficient to alter BK channel gating across this phylogenetic spectrum (Chu and Treistman, 1997; Jakab et al., 1997; Dopico et al., 1998; Brodie and Appel, 2000; Walters et al., 2000; Dopico, 2003). The constitutive pore-forming $\alpha$ subunit is the minimal unit required for ethanol modulation of the BK channel (Brodie et al., 2007). The channel's probability of opening $P_{o}$, probability of

The authors declare that they have no conflicts of interest with the contents of this article.

This research was supported by the National Institutes of Health National Institute on Alcohol Abuse and Alcoholism [Grant AA020992], donations from Tom Calhoon, and HornRaiser Crowdfunding.

https://doi.org/10.1124/jpet.118.251918.

S This article has supplemental material available at jpet.aspetjournals.org. opening, changes within minutes after ethanol exposure. However, whether the $\mathrm{P}_{\mathrm{o}}$ goes up or down, either transiently or more persistently, depends upon regulatory subunit expression, post-translational modifications, and the channel's microenvironment (Dopico et al., 2016).

Expressed in multiple neuronal compartments, the BK channel is well-positioned to broadly mediate the effects of ethanol on central nervous system (CNS) function (Wang et al., 2001; Dopico et al., 2014; Alqadah et al., 2016). In Caenorhabditis elegans and Drosophila, genetic manipulation of the highly conserved $\mathrm{BK} \alpha$ channel influences alcoholrelated behaviors; null mutations reduce acute ethanol intoxication and tolerance (Davies et al., 2003; Ghezzi and Atkinson, 2011), and overexpression reduces ethanol withdrawal severity (Scott et al., 2017b). Similarly, genetic manipulations that alter BK channel function in rodents influence ethanol tolerance and consumption (Martin et al., 2008; Kreifeldt et al., 2013), and a gain-of-function mutation increases acute responses to alcohol in humans (Du et al., 2005).

Genetic approaches to test the in vivo importance of this molecular target of ethanol have proved informative. Behavioral genetic and electrophysiological screening of $\mathrm{BK} \alpha$ 
channel mutants has yielded several mutations that restrict ethanol modulation of $\mathrm{BK} \alpha$ channel gating (Bukiya et al., 2014; Davis et al., 2014). We recently identified a BK channel mutant that exhibited limited ethanol modulation in vitro, and prevented BK channel-mediated acute ethanol intoxication in vivo. Restricted ethanol responses occurred without gross changes to baseline gating or other BK channel-dependent behaviors (Davis et al., 2014). Nevertheless, genetic approaches may be limited by changes in nervous system function caused directly by lifelong alteration of a molecule and/or indirectly via mechanisms compensating for the molecular alteration. BK channel deletion in mice results in obvious CNS and neuromuscular abnormalities (Meredith et al., 2004; Rüttiger et al., 2004), which can obscure the interpretation of alcohol-related behavioral effects. Even without widespread defects in basal physiology, one cannot disentangle the direct effect of manipulating the genetic target from the influence of potential compensatory changes. For these reasons, pharmacological approaches complement genetic approaches to address the in vivo importance of putative ethanol targets. Pharmacological agents can be administered during a circumscribed period, and are particularly powerful if they selectively occlude the functional impact of ethanol on the target molecule. Agents with this pharmacological action are lacking for most ethanol targets, including the BK channel.

To expand our toolset for understanding the action of ethanol at the BK channel, we developed a screen to search for pharmacological agents that restricted the BK channel's physiologic response to ethanol with little impact on basal channel function. Phage display for ion channels, pioneered by Tipps et al. (2010), allows a high-throughput screen for target binding. Although not as high-throughput, phenotypic screens can select agents with functional activity at a specific molecular target (Kwok et al., 2006) or a specific action, e.g., neuroprotection (Mondal et al., 2018) or antagonizing fetal alcohol syndrome (Wilkemeyer et al., 2003). Previously, we combined phage display and a secondary phenotypic screen in worm to identify a high-affinity modulator of BK channel function (Scott et al., 2017a). In the present study, our challenge was to select a modulator of the effects of another pharmacological agent at a specific target. Our high-throughput peptideidentification screen and a worm assay for rapid functional screening identified novel peptides that antagonized ethanol effects at the BK channel. One novel 9-amino acid peptide, LS10, acted specifically at the BK channel to reduce acute ethanol intoxication in wild-type (WT) and BK channel-humanized C. elegans strains. LS10 also restricted ethanol-induced changes in human $\mathrm{BK} \alpha$ channel gating in vitro with limited impact on basal function. These findings provide proof-of-concept evidence that a combined phagemid-display/behavioral genetics screening approach provides novel tools like LS10 to further our understanding of how alcohol acts at the BK channel to exert influence over CNS function.

\section{Materials and Methods}

Peptide Selection and Synthesis. A monovalent phagemiddisplay library (library C; Mobitec, Goettingen, Germany) was panned against three sets of HEK293 cells expressing one of three ion channels. Two sets were for negative selection. For one set, cells were transfected (Lipofectamine 2000; Invitrogen) with hGlyR $\alpha 1$ (X52009) and used 48 hours later. Another line stably expressed the rat small conductance calcium-activated channel 2 (U69882.1). For positive selection, cells stably expressed the human $\mathrm{BK} \alpha$ channel ZERO isoform (NM_002238). Phagemid particles obtained by superinfection were purified and diluted to a titer of $10^{11}$ to $10^{12}$ per milliliter using standard procedures (Röttgen and Collins, 1995). One milliliter of this solution was serially incubated with each set of HEK293 cells for 45 minutes. For the negative selection plates, the supernatant was collected and transferred to the next plate. After incubation with the positive selection plate, the cells were rinsed three times and the phage was eluted at a $\mathrm{pH}$ of 2.2. The progress of the phagemid selection process was monitored after each of five panning rounds by titering and sequencing the phagemid DNA. The selected peptide sequences were then synthesized as trifluoroacetic acid salts at 98\%-99\% purity verified by high-performance liquid chromatography and mass spectrometry analysis (Genscript, Piscataway, $\mathrm{NJ}$ ). Stocks were dissolved in water at $10 \mathrm{mM}$ and aliquots were lyophilized and stored at $-80^{\circ} \mathrm{C}$. The concentration of these aliquots was verified by disulfide bond UV absorption at $280 \mathrm{nM}$ (Beckman spectrophotometer Beckman Coulter (Brea, CA)). As a secondary determination of identity and purity of LS10, in-house liquid chromatography/mass spectrometry was performed on a single quadrupole mass spectrophotometer (6130; Agilent, Santa Clara, CA) interfaced with high-performance liquid chromatography with a diode-array (UV-visible) detector (1200; Agilent).

C. elegans Strains. Worms were maintained at $20^{\circ} \mathrm{C}$ on standard 6-cm diameter Petri dishes filled with 12-ml nematode growth media/agar and seeded with OP50 bacteria as described previously (Brenner, 1974). Worms cultured on plates contaminated with fungi or other bacteria were excluded from this study. The reference WT strain was N2 Bristol. The slo-1 null strain was NM1968, harboring the previously characterized null allele, js379 (Wang et al., 2001). The transgenic human $\mathrm{BK} \alpha$ strain $[h S L O(+)]$ was JPS340, which expressed cDNA of the human $\mathrm{BK} \alpha$ channel ZERO isoform under the endogenous pslo-1 promoter on a slo-1(js379) background (Davis et al., 2014).

C. elegans Behavioral Assays. Ethanol plates (400 mM) were prepared at the start of the experiment by adding $280 \mu \mathrm{l}$ of 200 -proof ethanol (Sigma Aldrich, St. Louis) beneath the agar of a standard unseeded plate. The plates were sealed with Parafilm. Age-matched day 1 adults were cleaned of bacteria by crawling on an unseeded plate. The worms were then incubated in a puddle of vehicle (nematode growth media) or $750 \mu \mathrm{M}$ peptide on an unseeded plate. The puddle was refreshed one to two times as needed, but let to fully absorb into the agar by 30 minutes. Pharmaco-behavioral analyses in C. elegans requires high doses because of the difficulty in getting pharmacological agents across the worm cuticle; furthermore, the brief duration (30 minutes) and mode of treatment (swimming in liquid) allows for minimal ingestion (Bull et al., 2007). Crawl behavior was then videoed (Flea2 camera; Point Gray Research, Richmond, Canada; StreamPix 3; NorPix, Montreal, Canada). Copper rings were used to restrict movement to a proscribed area for the crawl videos. The worms were moved to ethanol plates, and after 20 minutes crawl behavior was recorded again. The worms were tracked offline using semiautomated custom macros (Image-Pro; MediaCybernetics, Rockville, MD) for 1 minute to obtain crawl speed (centimeter per minute) by an impartial observer blind to genotype and peptide treatment. Crawl speeds were normalized to the mean speed of vehicle-treated yoked controls run concurrently with peptide-treated worms in each condition (baseline or ethanol exposed). This controlled for drifts in behavior and/or conditions over the several year course of data collection. However, similar results were found with non-normalized data (Fig. 2 vs. Supplemental Table 1). Normalized group mean \pm S.E.M. values for the peptide-treated and nematode growth media-treated control groups run in tandem were compared with Student's $t$ tests. Selection criteria for each step of the screen are described in Results.

HEK293 Cell Maintenance and Transfection. HEK293 cells (ATCC, Manassas, VA) were grown according to standard procedures. Cells were cultured at $37^{\circ} \mathrm{C}$ in a $5 \% \mathrm{CO}_{2}$ atmosphere in Dulbecco's 
modified Eagle's medium with l-glutamine, sodium pyruvate, and 10\% fetal bovine serum (ThermoFisher Scientific). Cell lines were split with trypsin/EDTA in Hanks' balanced salt solution (ThermoFisher Scientific) up to 25-30 cycles. For electrophysiological recordings, cells were transfected (Polyfect; Qiagen Hilden, Germany) with the human $\mathrm{BK} \alpha$ (hSLO) ZERO or STREX isoform. Enhanced green fluorescent protein was cotransfected as a marker. Electrophysiological recordings were made 16-72 hours after transfection.

Xenopus Oocyte Expression. SLO-1 was expressed heterologously in oocytes because the channel did not express at detectable levels in HEK293 cells. To obtain Xenopus laevis oocytes, frogs (Nasco, Fort Atkinson, WI) were anesthetized with tricaine, and portions of their ovaries were removed surgically in accordance with the Association for Assessment and Accreditation of Laboratory Animal Care regulations (National Research Council, 2011). A plasmid containing the coding sequence for the slo-1a variant, mg180 (plasmid \#34660; Addgene, Cambridge, MA), was a gift from M. Goodman (Johnson et al., 2011). The slo-1a variant was chosen because it is the most abundantly expressed in worm (Glauser et al., 2011), and expression of this variant is sufficient to rescue acute behavioral intoxication to ethanol in slo-1 null worms (Davis et al., 2014). This plasmid was linearized with $\mathrm{XbaI}$ and transcribed in vitro (mMessage mMachine kit; Life Technologies). Oocytes were injected with $5 \mathrm{ng}$ of capped RNA and then stored individually at room temperature in modified Barth's solution.

Patch-Clamp Recordings. Voltage-clamp recordings were performed at room temperature $\left(22-24^{\circ} \mathrm{C}\right)$ using an inside-out configuration. For all recordings, the extracellular solution contained the following: $2 \mathrm{mM} \mathrm{KCl}, 136 \mathrm{mM} \mathrm{KOH}, 20 \mathrm{mM}$ Hepes, and $2 \mathrm{mM} \mathrm{MgCl}_{2}$, adjusted to $\mathrm{pH} 7.2$ with $\mathrm{MeSO}_{3} \mathrm{H}$. To apply peptide to the extracellular surface, patch electrodes (7-20 M $\Omega$ in resistance) were tip filled with normal extracellular solution and backfilled with extracellular solution containing $500 \mathrm{nM}$ LS10. Enough normal extracellular solution was included to provide about 10 minutes of peptide-free recording (Scott et al., 2017b). The intracellular solution contained the following: $6 \mathrm{mM} \mathrm{KCl}, 132 \mathrm{mM} \mathrm{KOH}$, and $20 \mathrm{mM}$ Hepes, adjusted to $\mathrm{pH} 7.2$ with $\mathrm{MeSO}_{3} \mathrm{H}$. For hSLO recordings without ethanol, $\sim 750 \mathrm{nM}$ free $\mathrm{Ca}^{2+}$ was achieved with $4.17 \mathrm{mM} \mathrm{CaCl}_{2}$ and $5 \mathrm{mM}$ EGTA, a ratio verified by measurement with a $\mathrm{Ca}^{2+}$-sensitive electrode. For hSLO recordings with ethanol, $2 \mathrm{mM} \mathrm{MgCl}_{2}, 3.85 \mathrm{mM} \mathrm{CaCl}_{2}$, and $5 \mathrm{mM}$ EGTA were added for an estimated $638 \mathrm{nM}$ free $\mathrm{Ca}^{2+}$ (MaxChelator). For all SLO-1 recordings, the internal solution contained $\sim 5 \mu \mathrm{M}$ free $\mathrm{Ca}^{2+}$ achieved with $2.644 \mathrm{mM} \mathrm{CaCl}_{2}$ and $5 \mathrm{mM}$ HEDTA, a ratio verified by measurement with a $\mathrm{Ca}^{2+}$-sensitive electrode. For recordings with ethanol treatment, $50 \mathrm{mM}$ ethanol was added to the bath for intracellular application.

Voltage-clamp recordings were made with an Axopatch 200A amplifier (10 kHz filtering; Molecular Devices San Jose, CA) at $50 \mathrm{kHz}$ sampling using custom macros in IgorPro (Wavemetrics, Portland, OR) or Patchmaster (HEKA; Lambrecht, Germany). The measurement of the $\mathrm{P}_{\mathrm{o}}$ values for each experiment is detailed in Fig. 5 and Supplemental Figs. 2 and 3. Group comparisons for the mean \pm S.E.M. values were made using planned paired or unpaired Student's $t$ tests and two-way repeated measures analysis of variance with Holm-Sidak post-hoc correction as stated in the figure legends (Figures 2-5, Supplemental Figures 2 and 3).

\section{Results}

Phagemid-Display Screen for BK Channel-Binding Peptides Selects Unique Sequence Motifs. To screen for small peptides that modulate the BK channel's physiologic response to ethanol, we first performed a high-throughput phagemid-display screen designed to enrich BK channelbinding peptides. The phagemid library was comprised of approximately $3 \times 10^{7}$ peptide sequences generated on a cyclic, 9-amino acid scaffold with six randomized positions.
For positive selection, the phagemid library was panned against HEK293 cells expressing the ZERO isoform of the human $\mathrm{BK} \alpha$ channel (hSLO). The ZERO isoform was chosen because it is widely expressed in the mammalian nervous system and is sensitive to ethanol (Pietrzykowski et al., 2008; Dopico et al., 2016). For negative selection, the library was screened against another ethanol target protein, the glycine receptor (hGlyR $\alpha 1$ ) as well as another calcium-sensitive potassium channel (rSK2) (Fig. 1A). Positively charged residues were enriched in identified peptide sequences, particularly arginine, as were the small amino acids glycine and alanine (Fig. 1B). The sequences were further analyzed to determine whether the panning procedure enriched certain sequence motifs. A sequence motif was defined as two or more amino acids in a fixed position, or three or more amino acids in a sliding position. Among the 20 peptides derived from our screen (Table 1), 13 sequence motifs were enriched 150- to $\sim 60,000$-fold over their expected frequencies in the original library (Fig. 1C). A previous study from our laboratory characterized the ethanol-independent effects of the single peptide LS3 (Scott et al., 2017a). Here, we present a detailed analysis of all 20 peptides (LS1-LS20) in the presence and absence of ethanol.

C. elegans Behavioral Screen Selects Peptides with BK Channel- and Ethanol-Dependent Activity In Vivo. We synthesized the 20 peptides and screened them for functional activity at the BK channel using a $C$. elegans phenotypic assay. C. elegans expresses a highly conserved BK channel ortholog, SLO-1 (Sonnhammer and Durbin, 1997; Lai et al., 2000). SLO-1 is one of the many gene products that modulate the C. elegans neuromuscular circuitry to control crawling locomotion (Wang et al., 2001). Moreover, SLO-1 plays a key role in mediating the locomotor effects of acute ethanol intoxication in worms (Davies et al., 2003). To probe for peptide-induced changes in locomotion, age-matched, day 1 adult WT worms were preincubated with either peptide or vehicle for 30 minutes. Crawl speed was then measured before and after 20-minute exposure to ethanol. This acute ethanol exposure leads to an internal concentration of $\sim 40 \mathrm{mM}$ ethanol (Scott et al., 2017b), a pharmacologically relevant concentration for ethanol intoxication (Dopico et al., 2014). Intoxication was observed as a $\sim 45 \%$ reduction in crawl speed for vehicle-treated WT worms [crawl speed before and after ethanol exposure, respectively: $0.92 \pm 0.011$ and $0.51 \pm 0.011 \mathrm{~cm} / \mathrm{min}$; $t(1953)=26.05, P<0.001]$. To ascertain whether peptide treatment altered crawl speed at baseline or in response to acute ethanol exposure, the crawl speeds of peptide-treated worms were compared with the speeds of vehicle-treated yoked controls tested concurrently. Half of the peptides caused a significant (set at $P<0.001$ ) change in the crawl speed of WT worms at baseline (Fig. 2A) and/or after ethanol exposure (Fig. 2B). Five more peptides showed a trend toward altering the crawl speed of WT worms in either condition (set at $P<0.05$ ). Finally, five peptides, LS15-LS18 and LS20, showed no effects on WT crawl speed in either condition and were eliminated from our screen (Fig. 2, C and $\mathrm{D}$, hatched boxes).

Next, we used a strain lacking the BK channel (slo-1 null) to determine whether the ethanol- and ethanol-independent locomotor effects of the 15 peptides remaining in the screen were mediated by changes in BK channel function. As for WT worms, slo-1 null worms were preincubated with either 

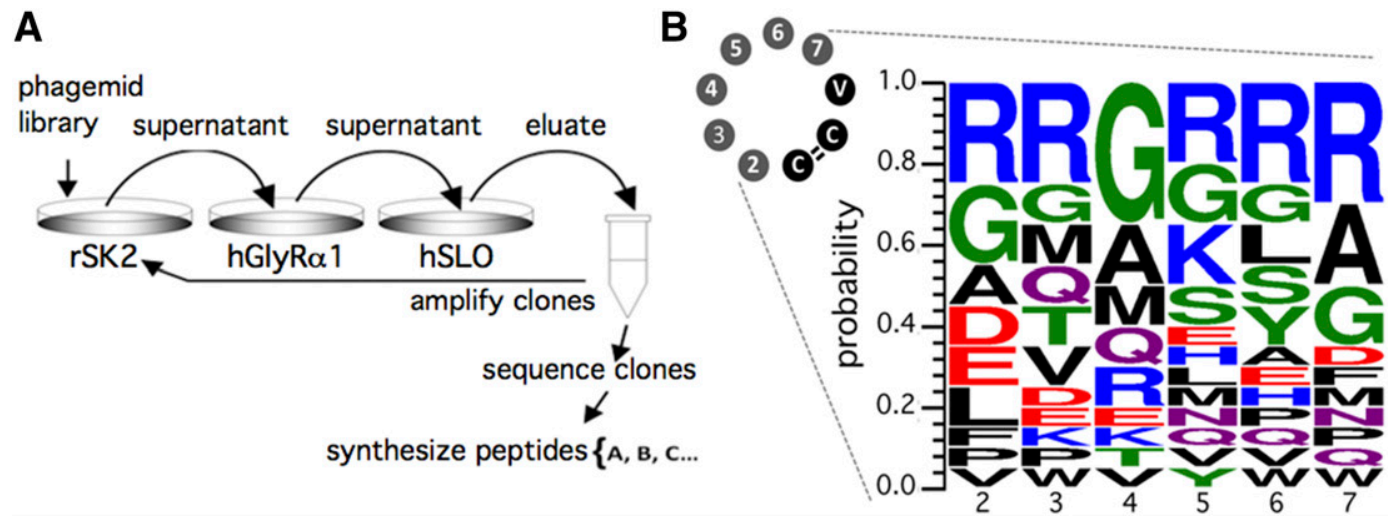

\begin{tabular}{lc} 
C & fold \\
motif & enrichment \\
\hline XRXXYR & 59259 \\
XXGRXA & 59259 \\
RRXXXX & 4444 \\
*GGGG & 3951 \\
XRGXXX & 2963 \\
RXXXXA & 2963 \\
XXGXLX & 2963 \\
GMXXXX & 2963 \\
XXXKRX & 2963 \\
XQXXXG & 2963 \\
DXMXXX & 2963 \\
*EQR & 148 \\
*GRR & 148
\end{tabular}

Fig. 1. Peptide motifs enriched by phagemid-display screen targeting the BK channel. (A) Phagemids displaying approximately 30 million unique peptide sequences were screened for their putative abilities to bind to the human BK $\alpha$ channel (hSLO). Phagemids were serially incubated with HEK293 cells expressing rat SK2 and human GlyR $\alpha 1$, respectively, to remove nonspecific phagemid prior to incubation with HEK293 cells expressing hSLO. Phagemids were sequenced after rounds 3-5 of panning to reveal 26 remaining peptide sequences. (B) Sequence logo showing the relative likelihood of amino acid expression for all 20 candidate peptides selected by the phagemid-display screen (see Table 1). Only the residues that were randomized in the phagemid-display library (amino acids 2-7) are shown. The larger the letter denoting the amino acid, the more prevalent that amino acid was in the identified peptides. Charged amino acids, particularly arginine $(R)$, were highly enriched at all positions followed by small amino acids like glycine (G) and alanine (A). (C) Two or more amino acids in a fixed position or three or more amino acids in a sliding position (*) were considered motifs. Motifs are shaded according to fold enrichment over theoretical frequency in the original phagemid-display library.

peptide or vehicle for 30 minutes and then crawl speed was measured before and after 20-minute exposure to ethanol. Consistent with previous findings (Davies et al., 2003), vehicle-treated worms carrying the slo- 1 null allele, $j$ s379, were largely resistant to the intoxicating effects of ethanol, showing only a $\sim 10 \%$ reduction in crawl speed $[0.52 \pm 0.011$ and $0.45 \pm 0.0090 \mathrm{~cm} / \mathrm{min}$ before and after ethanol exposure, respectively; $t(1546)=4.46, P<0.001]$. The crawl speeds of peptide-treated worms were compared with the speeds of vehicle-treated yoked controls tested concurrently. Of the 15 peptides that showed strongly significant effects on WT crawl speed, only five peptides showed no effect (set at $P>0.05$ ) on slo-1 null mutant crawl speed either before or after ethanol exposure (Fig. 2, C and D). These five peptides, LS3, LS10, LS11, LS13, and LS19 (Fig. 2, turquoise bars; Supplemental Table 1), were designated as having BK channel-dependent physiological activity; i.e., they likely altered C. elegans locomotor behavior by specific modulation of worm BK channel function. The other 10 peptides that modified crawl speed in slo- 1 null mutant worms may act on additional nonspecific targets and were thus eliminated from further analysis (Fig. 2, C and D, gray bars).

We hypothesized that the five selected peptides would also alter the physiological activity of the human BK channel due to 1) conservation of the channel and 2) the use of the human channel during positive selection in our phagemid-display screen. To test this hypothesis we used a BK channel-humanized worm strain $[h S L O(+)]$ expressing the same human BK channel isoform as for phagemid display (ZERO) in the slo-1 null mutant background. Similar to WT worms, acute ethanol intoxication caused a $\sim 40 \%$ reduction in crawl speed for vehicle-treated humanized worms [crawl speed before and after ethanol exposure: $0.60 \pm 0.019$ and $0.38 \pm 0.012 \mathrm{~cm} / \mathrm{min}$, respectively; $t(1066)=8.30, P<0.001]$. Thus, as we previously reported (Davis et al., 2014) the human BK channel can functionally substitute for the endogenous SLO-1 channel for ethanol intoxication.
Four out of five of the peptides that affected worm BK channel function in WT worms also caused a significant change in crawl speed before and/or after ethanol exposure for the humanized $h S L O(+)$ strain (Fig. 3, A and B, turquoise bars; Supplemental Table 1). Although they do not share any potential amino acid sequence motifs (Fig. 3C; Table 1), two peptides, LS3 and LS10, restricted the sedative effects of acute ethanol exposure [crawl speed before and after ethanol exposure: for the LS3-treated peptide, $0.34 \pm 0.021$ and $0.45 \pm 0.027 \mathrm{~cm} / \mathrm{min}, t(173)=3.2, P<$ 0.01; and for the LS10-treated peptide, $0.62 \pm 0.040$ and $0.54 \pm$ $0.053 \mathrm{~cm} / \mathrm{min}, t(174)=1.1$, N.S.]. LS3 slowed baseline crawl speed for both WT and humanized worms (Scott et al., 2017a). Thus, we focused on investigating LS10 because the peptide limited the physiologic effects of ethanol without significantly altering the basal BK channel function in the humanized strain.

Peptide LS10 Restricts Ethanol-Induced Changes in Gating for Heterologous BK $\boldsymbol{\alpha}$ Channels. To observe the action of peptide LS10 on worm and human BK channel gating

TABLE 1

Peptide sequences selected by phagemid display

\begin{tabular}{lcc}
\hline Name & Sequence & Enriched Motif \\
\hline LS1 & CARGVYRVC & XRXXYR and XRGXXX \\
LS2 & CRVAHRAVC & RXXXXA \\
LS3 & CRRGLVQVC & XRGXXX and RRXXXX \\
LS4 & CPPGRGAVC & XXGRXA \\
LS5 & CGMTKRPVC & GMXXXX and XXXKRX \\
LS6 & CDEMNWWVC & DXMXXX \\
LS7 & CERRMYRVC & XRXXYR \\
LS8 & CRRAYEMVC & RRXXXX \\
LS9 & CRRKRHAVC & XXGRXA and XXXXXI \\
LS10 & CAVGRLAVC & EQR and XQXXXG \\
LS11 & CLQEQRGVC & GRR \\
LS12 & CRKQGRRVC & GRR \\
LS13 & CEGRRARVC & XXGXLX \\
LS14 & CLDGKLDVC & GGGG \\
LS15 & CGGGGSRVC & GGGG \\
LS16 & CFTGGGGVC & GXXKXX \\
LS17 & CVWVKRNVC & GMXXX \\
LS18 & CGMASSFVC & XQR and DXMXXX \\
LS19 & CDTMEQRVC & CGQXX \\
LS20 & CGQQSPGVC & \\
\hline
\end{tabular}


no ethanol

A

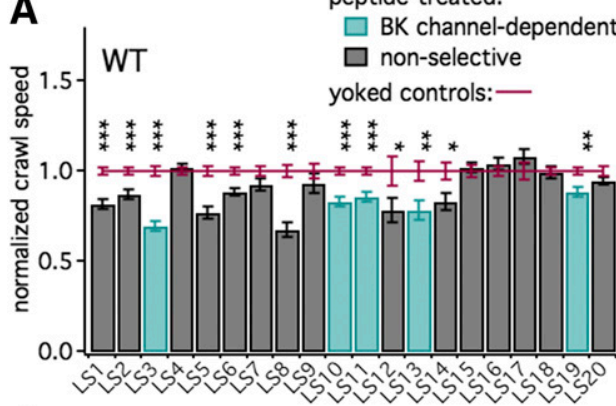

C

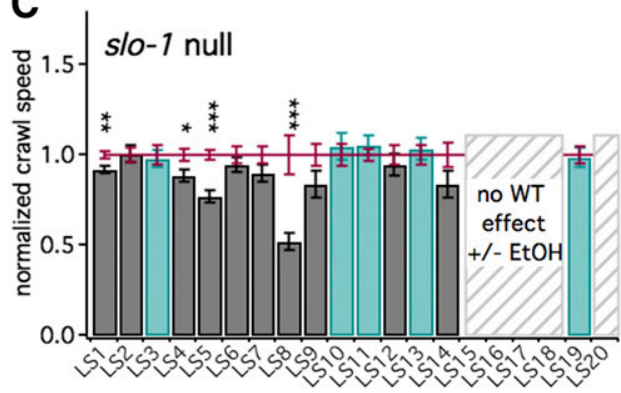

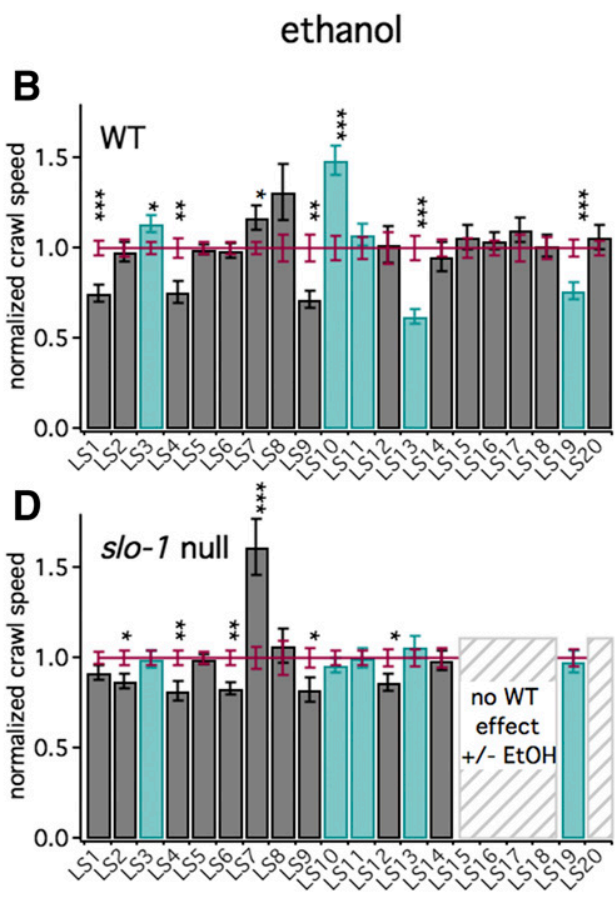

Fig. 2. Subset of peptides selectively altered behavior of C. elegans in a BK channel-dependent manner. Worms were preincubated with peptide (LS1-LS20) or vehicle, and then crawl speed was measured both before (no ethanol, left panels) and after (ethanol, right panels) acute exposure to ethanol. Crawl speeds were normalized to the mean speed of vehicle-treated yoked controls. Mean \pm S.E.M. values are shown as bars for the peptidetreated groups and as lines (maroon) for the vehicle-treated groups. (A) For WT worms, a subset of peptides decreased baseline crawl speed (normalized crawl speed $<1$ ). (B) For ethanol-exposed WT worms, a subset of peptides either enhanced (normalized crawl speed $<1$ ) or reduced (normalized crawl speed $>1$ ) ethanol-induced locomotor sedation. In all, 10 peptides significantly (set at $P<0.001$ ) altered WT crawl speed in the presence or absence of ethanol and were considered physiologically active. Five more were potentially physiologically active, showing a trend toward altered crawl speeds $(P<$ 0.05). Five peptides showed no effect on WT with or without ethanol and were eliminated from the screen. (C and D) Of the remaining 15 peptides, only five showed no effect (significance set at $P<0.05$ ) on baseline or ethanol-exposed crawl speeds for a strain lacking a functional BK channel (slo-1 null). These peptides with BK channel-dependent physiological activity are indicated by turquoise bars. Student's $t$ tests compared the peptide-treated vs. vehicle-treated worms run in tandem, $* * * P<0.001 ; * * P<0.01 ; * P<0.05$

in vitro, we made electrophysiological recordings of $\mathrm{BK} \alpha$ channels expressed heterologously. The ZERO and STREX isoforms of the human $\mathrm{BK} \alpha$ channel (hSLO-ZERO and hSLOSTREX, respectively) were expressed in HEK293 cells, while the worm BK channel (SLO-1) was expressed in oocytes. Both were recorded in inside-out patches at $\sim 750 \mathrm{nM}$ free internal calcium for hSLO and $\sim 5 \mu \mathrm{M}$ for SLO-1. Higher internal calcium concentrations were used for SLO-1 because invertebrate channels show less activation at low calcium concentrations (Johnson et al., 2011). LS10 was applied via diffusion to the extracellular side of the patch. Neither hSLO isoform showed a net change in the $\mathrm{P}_{\mathrm{o}}$ across patches with application of 500 nM LS10 (Fig. 4B; Supplemental Fig. 2, A and B). By comparison, we found that the $\mathrm{P}_{\mathrm{o}}$ value of SLO-1 showed a consistent decrease by over half in the presence of $500 \mathrm{nM}$ LS10 (Fig. 4C; Supplemental Fig. 2C). Although measured outside the physiologic voltage range, the differential effects we observed for LS10 on the $\mathrm{P}_{\mathrm{o}}$ of SLO-1 and hSLO parallels the presence and absence of baseline behavioral effects in WT and humanized worms, respectively (Fig. 2A; Fig. 3A).

Because peptide LS10 suppressed the BK channel-mediated effects of acute intoxication in vivo, we predicted that LS10 would also alter the effects of ethanol on BK channel gating in vitro. To test this idea, we recorded hSLO-ZERO in insideout patches with a combination of high intracellular $\left[\mathrm{Mg}^{2+}\right]$ and low intracellular $\left[\mathrm{Ca}^{2+}\right]$. In control experiments, hSLOZERO displayed a highly reliable decrease in the $\mathrm{P}_{\mathrm{o}}$ several minutes after the intracellular application of $50 \mathrm{mM}$ ethanol
(Fig. 5, A-C; Supplemental Fig. 3B). Similar to our findings in the absence of high intracellular $\left[\mathrm{Mg}^{2+}\right]$, preincubation of the patch ( 15 minutes) with 500 nM LS10 did not consistently alter the baseline $P_{o}$ at $20-60 \mathrm{mV}$ (Supplemental Fig. 3A). In contrast, preincubation with LS10 abolished the ethanol-induced decrease in the $\mathrm{P}_{\mathrm{o}}$ (Fig. 5, A-C; Supplemental Fig. 3B). In a small set of inside-out patches, we also tested the effect of LS10 on worm BK channels. SLO-1 expressed in oocytes was recorded under the same conditions described previously. Intracellular application of $50 \mathrm{mM}$ ethanol caused a transient increase in the SLO-1 $\mathrm{P}_{\mathrm{o}}$, which was limited by preincubation with $500 \mathrm{nM}$ LS10 (Fig. 5, D-F). Together, these findings demonstrate that peptide LS10 restricts changes in $\mathrm{BK} \alpha$ channel gating in response to acute ethanol exposure.

\section{Discussion}

From worms to humans, the BK channel appears to be a conserved target of alcohol. Tools for understanding the behavioral consequence of alcohol's action at a single molecular target, like the BK channel, would ideally modulate the target's response to ethanol without altering baseline function. In the present study we developed a screening paradigm to search for pharmacological agents that restrict the BK channel's response to ethanol with limited impact on basal channel function. Using this screening paradigm, we report the identification and characterization of a small peptide, 

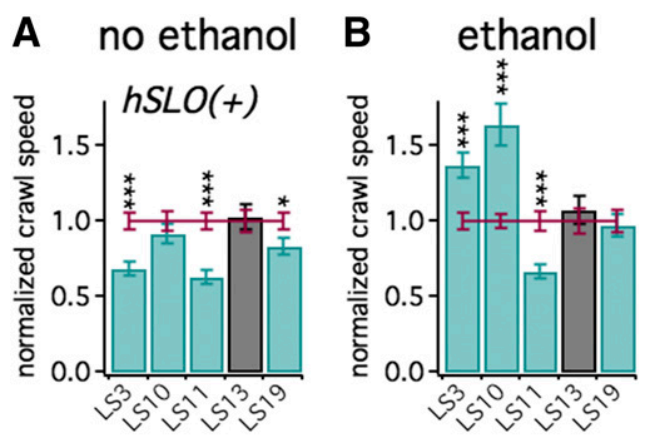

peptide-treated: $\square$ BK channel-dependent yoked controls: $\square$ non-selective

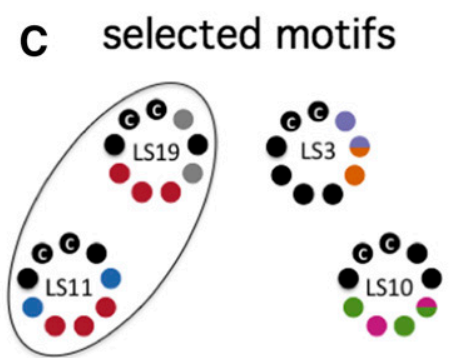

Fig. 3. Peptides altered behavior of BK channel-humanized worms. (A and B) Day 1 adult worms expressing the human $\mathrm{BK} \alpha$ channel $[h S L O(+)]$ in a slo-1-null background were preincubated with peptide or vehicle. Crawl speed was then assessed before (A) and after acute exposure to ethanol (B). Four peptides showed significant $(P<0.05)$ effects (turquoise). Crawl speeds were normalized to the vehicle-treated yoked controls where 1.0 signifies basal speed (A) and intoxicated speed (B), respectively. Mean \pm S.E.M. values are shown as bars for the peptidetreated groups and lines (maroon) for the vehicle-treated groups. Student's $t$ tests compared peptide-treated vs. vehicle-treated worms run in tandem, $* * * P<0.001 ; * P<0.05 ; N>60$. (C) Schematic illustration representing the enriched amino acid motifs in the four selected peptides. Each motif is represented by a unique color. Dual coloring indicates the residue is shared between motifs. LS19 and LS11 peptides share a motif.

LS10, which restricts the effects of ethanol at the $\mathrm{BK} \alpha$ channel in vitro and in vivo. Electrophysiological recordings showed that LS10 suppressed ethanol modulation of human $\mathrm{BK} \alpha$ channel gating in the submicromolar concentration range, with limited effects on basal channel function. In both WT and BK channel-humanized $C$. elegans, LS10 reduced acute ethanol intoxication via a BK channel-dependent mechanism. Notably, LS10 did not substantially affect baseline behavior of the humanized strain. Together, our findings indicate that our screening paradigm will be useful for developing tools like LS10 to study BK channel modulation by ethanol and how this contributes to alcohol-related behaviors.

To select a peptide that limited ethanol modulation without substantial effects on basal $\mathrm{BK} \alpha$ channel function, we harnessed the power of a high-throughput phagemid-display assay to screen millions of peptide sequences against the human $\mathrm{BK} \alpha$ channel. We then secondarily screened for BK channel- and ethanol-dependent physiological activity, capitalizing on the importance of the worm BK channel for both acute ethanol intoxication and baseline locomotion (Davies et al., 2003). This screen succeeded in selecting a peptide that alters ethanol modification of $\mathrm{BK} \alpha$ channel gating. We found that LS10 restricted acute intoxication for WT and humanized worms and limited ethanol-dependent changes in gating for both human and worm $\mathrm{BK} \alpha$ channels in vitro. Moreover, eliminating peptides that caused locomotor slowing in ethanol naive worms successfully eliminated peptides that substantially altered baseline $\mathrm{BK} \alpha$ channel function in vitro. Gainand loss-of-function BK channel mutations both slow baseline locomotion in worm (Davies et al., 2003), and several peptides screened here caused BK channel-dependent reductions in baseline locomotion for WT and BK channel-humanized worms. We previously reported that one of these, LS3, altered the basal probability of opening of the human $\mathrm{BK} \alpha$ channel in vitro (Scott et al., 2017a). Here, we report that LS10 changed baseline behavior for WT but not humanized worms, corresponding to a reduction in basal probability of opening for the worm but not the human $\mathrm{BK} \alpha$ channel. Together, these findings support the ability of BK channel-dependent behavioral effects in worm to predict the effect of peptide treatment on BK channel gating in vitro. Moreover, the differential effects of LS10 on worm and human BK channel gating are not unexpected. Without splice inserts, the percent identity for these channels is over $60 \%$ from $\mathrm{S} 1$ through the calcium bowl, with even greater identity in key functional regions like the transmembrane and pore domains, RCK1, RCK2, and the calcium bowl. Nonetheless, there are striking differences in gating kinetics and activation by intracellular calcium for worm and human BK channels (e.g., see Fig. 3) that are likely to contribute to different responses to a modulator, even if it binds to an identical or highly similar pocket.

This screening technique could provide powerful tools, like LS10, for understanding ethanol's action at the BK channel. The putative ethanol-binding pocket identified for the mammalian BK channel (Bukiya et al., 2014), and another wellconserved ethanol-sensitive residue found in both the worm and human BK channels (Davis et al., 2014) reside on the channel's long intracellular cytoplasmic domain. LS10, applied extracellularly, may restrict ethanol modulation of $\mathrm{BK}$ channel gating via long-range allosteric interactions with the intracellular ethanol-binding site. Binding of ethanol to the BK channel potentiates or inhibits channel gating depending upon channel and cellular conditions, including ionic concentrations (Dopico et al., 2014). The recording conditions used herein for the human $\mathrm{BK} \alpha$ channel obtained a very consistent, stable response to ethanol, which was especially important given the greater fluctuation in the probability of opening for LS10- and ethanol-treated patches (Supplemental Fig. 3B). Together with the worm BK channel recordings, our data suggest that LS10 may inhibit ethanol binding or signal transduction of the event. As such, fully probing the biophysical mechanism through which LS10 influences ethanol modulation could provide support for the location of the ethanol binding pocket or elucidate the signal transduction pathway from ethanol binding to channel gating.

Peptides hold a prominent position in ion channel pharmacology. For the BK channel, peptide toxins predominantly derived from scorpion venom block the pore with high affinity and specificity but have relatively complex structures that impede large-scale synthesis and efficient blood-brain barrier permeability (Yu et al., 2016). Small, endogenous peptides or peptide fragments modulate BK channel function, but mainly act with lower affinity (White et al., 1991; Wallner et al., 1999; Xia et al., 2003; Zhang et al., 2014). Recently, we have introduced this new class of BK channel-modulating peptides, small 9-amino acid peptides not known to be found in nature (Scott et al., 2017a). The shared scaffold of these peptides, 
A

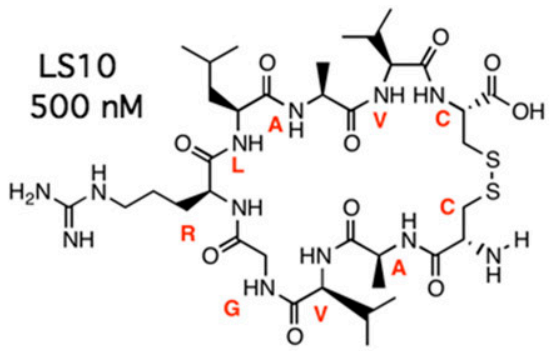

B

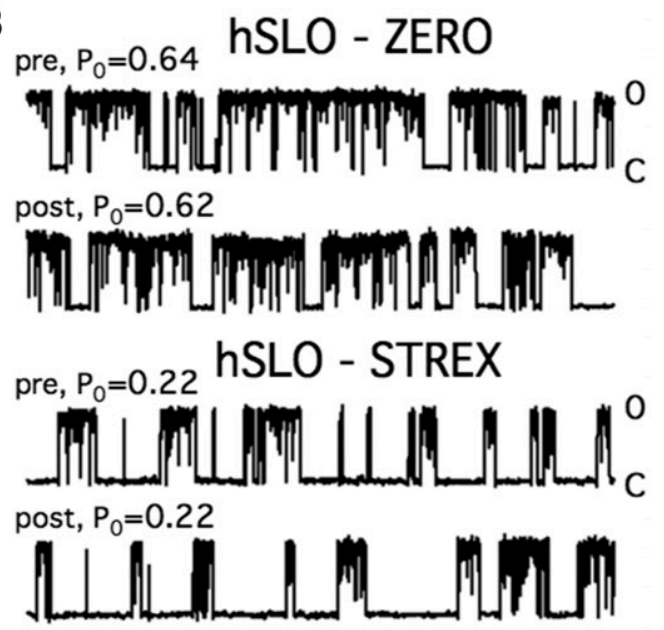

C

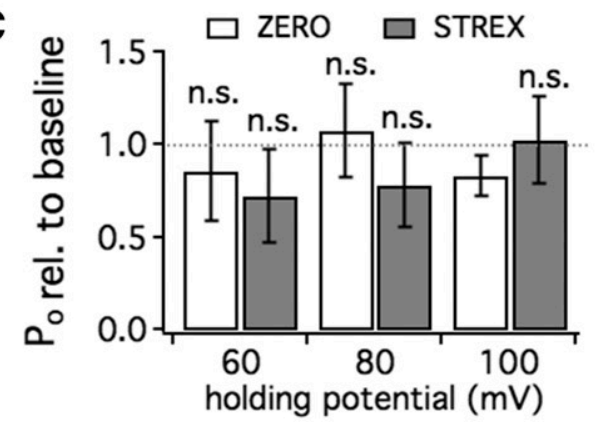

D

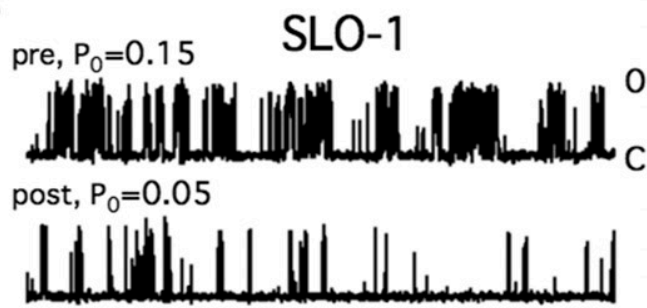

$\mathbf{E}$

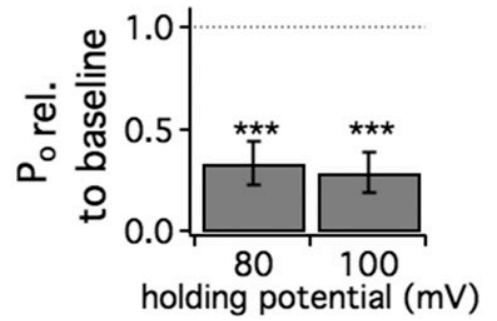

Fig. 4. Peptide LS10 substantially reduces the probability of opening of the worm BK channel but not the human BK channel. (A) Structure of LS10. (B) Representative single-channel recording (1 second) at $100 \mathrm{mV}$ before and after LS10 (500 nM) was applied by diffusion to the extracellular face of ZERO (upper traces) or STREX (lower traces) isoforms of the human $\mathrm{BK} \alpha$ channel (hSLO). (C) There was no significant change in $\mathrm{P}_{\mathrm{o}}$ in response to LS10 application for the ZERO (open bars) or STREX (shaded bars) isoforms. The $\mathrm{P}_{\mathrm{o}}$ was measured using five or more 3-second traces chosen for scalable synthesis and resistance to proteolytic degradation (Gudmundsson et al., 1999; Andersson et al., 2000), allows them to be developed for large-scale applications. We previously reported that one such peptide, LS3, acts with nanomolar efficacy and high specificity at the BK channel, modulating sound-evoked neural activity in the auditory midbrain (Scott et al., 2017a). LS10 shares a similar structure, with an $\mathrm{N}$ - to $\mathrm{C}$-terminal disulfide bridge and the presence of arginine, as well as submicromolar efficacy at the $\mathrm{BK} \alpha$ channel in vitro. Thus, like LS3, LS10 may exhibit CNS bioavailability in vivo without further modifications (Scott et al., 2017a).

Once in the brain, peptides like LS10 could be used to explore how ethanol modulation of BK channel function mediates alcohol-related phenotypes, such as acute intoxication. Knockout and knockin mutations, most of which rely on alterations to basal protein function, have made substantial contributions to our understanding of the functional impact of ethanol's action at individual proteins (Blednov et al., 2012; Borghese et al., 2012; Liu et al., 2013; Howard et al., 2014). A pharmacological tool has the advantage of manipulating the impact of acute ethanol administration at a target protein while eliminating the influence of even moderate compensatory mechanisms provoked by genetic mutations. LS10 limits the acute effects of ethanol on the ZERO isoform of the human $\mathrm{BK}$ channel function in vivo and in vitro. The $\mathrm{ZERO}$ isoform of the $\mathrm{BK}$ channel is nearly identical in rodents and humans and is the most commonly expressed isoform in brain (Chen et al., 2005). Moreover, the specificity of action at the BK channel in worm probed by our selection techniques may carry over to mammals since $C$. elegans homologs have been identified for many human genes (C. elegans Sequencing Consortium, 1998). While restricting acute ethanol modulation of the BK channel may not fully eliminate all aspects of acute ethanol intoxication due to the multitarget nature of ethanol, we predict that treatment with an LS10-like peptide will reduce at least some of the consequences of ethanol intake, particularly locomotor sedation. Interestingly, a structurally unrelated 9-amino acid peptide restricts ethanol-induced developmental toxicity in mouse (Wilkemeyer et al., 2003). Together, these findings suggest that peptides like LS10, and our recently identified BK channel mutation that restricts ethanol modulation (Davis et al., 2014), may play complementary roles in supporting our understanding of how BK channel modulation by ethanol contributes to alcohol-related behaviors.

The screening paradigm used to select LS10 may be able to further expand the pharmacological toolbox for dissecting the influence of BK channel subsets in acute intoxication. Modulation of BK channel function by acute ethanol application is influenced by $\mathrm{BK} \alpha$ splice variation, auxiliary subunit expression, and phosphorylation state (Dopico et al., 2014). For example, STREX isoforms show little functional modulation by ethanol while ZERO- and ALCOREX-containing isoforms show increasing levels of modulation, respectively

recorded over a duration of $\sim 5$ minutes. Postpeptide (post) $\mathrm{P}_{\mathrm{o}}$ plotted relative to baseline [prepeptide (pre)]. Pre vs. post, paired Student's $t$ tests, N.S.; $N=6-10$. (D) Representative single-channel recording (1 second) at $100 \mathrm{mV}$ before and after LS10 (500 nM) was applied by diffusion to the extracellular face of the worm BK channel (SLO-1). (E) The $\mathrm{P}_{\mathrm{o}}$ decreased in response to LS10 for SLO-1. Pre vs. post, paired Student's $t$ tests, $* * * P<0.005, N=8$. 
A

hSLO - ZERO

control

before ethanol, $\mathrm{P}_{0}=0.46$

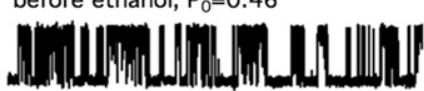

after ethanol, $\mathrm{P}_{0}=0.22$

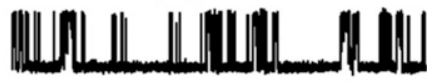

B

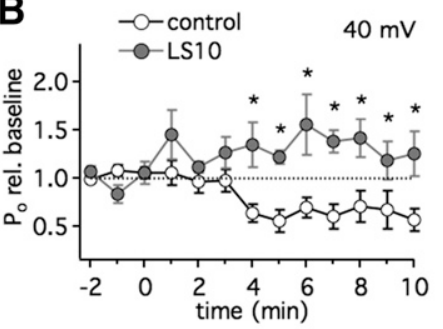

SLO-1

D

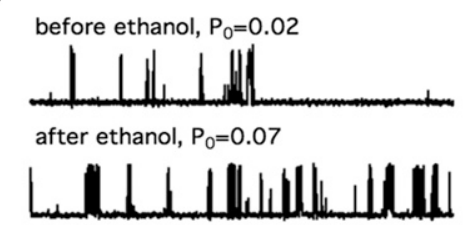

E

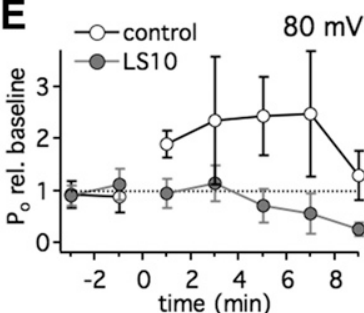

preincubated with LS10

before ethanol, $\mathrm{P}_{0}=0.43$

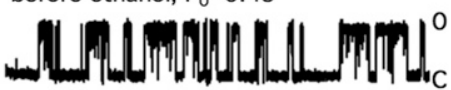

after ethanol, $\mathrm{P}_{0}=0.44$

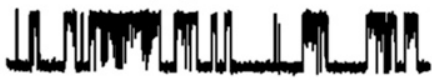

C $\square$ control

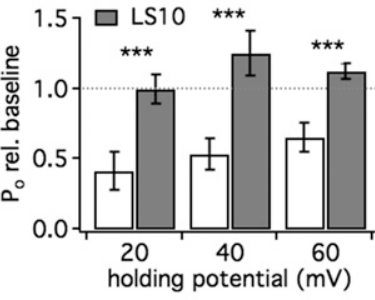

before ethanol, $\mathrm{P}_{0}=0.05$

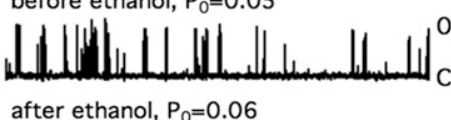

after ethanol, $\mathrm{P}_{0}=0.06$
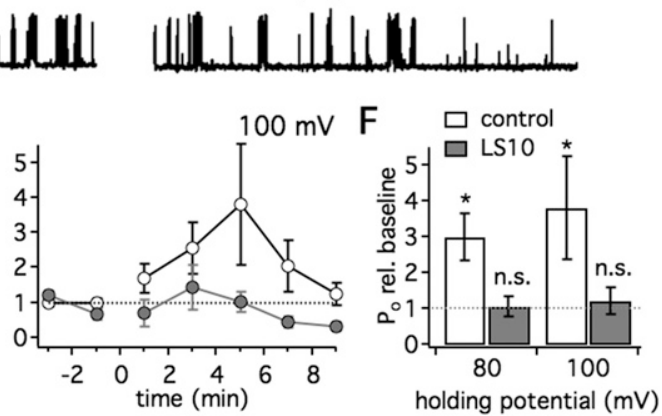

Fig. 5. Peptide LS10 limits changes in BK channel gating by acute ethanol exposure. (A) Representative singlechannel recording (3 seconds) of hSLO-ZERO expressed in HEK293 cells at $40 \mathrm{mV}$ before (upper traces) and after (lower traces) bath application of 50-mM ethanol. LS10 (500 $\mathrm{nM}$ ) was applied by diffusion to the extracellular face. (B) Control (open circles) recordings at $40 \mathrm{mV}$ showed a decrease in $\mathrm{P}_{0}$ relative to baseline after the application of ethanol (time $=0$ ) that was blocked by preincubation with 500 nM LS10 (shaded circles). The $\mathrm{P}_{\mathrm{o}}$ was measured each minute using three (3-second) traces and plotted relative to baseline as mean \pm S.E.M. Control vs. peptide, two-way repeated measures analysis of variance, post-hoc $P<0.05$, $N=7-10$. (C) The $\mathrm{P}_{\mathrm{o}}$ was significantly lower relative to baseline $\sim 10$ minutes after ethanol application for control recordings (open bars) than in the presence of LS10 (shaded bars). The $\mathrm{P}_{\mathrm{o}}$ was measured using 10 (3-second) traces recorded over a duration lasting several minutes. The $P_{0}$ value after ethanol is plotted relative to baseline. Ethanolinduced change in $\mathrm{P}_{\mathrm{o}}$ for control vs. peptide-treated, Student's $t$ tests, $* * * P<0.005, N=6-10$. (D) Representative single-channel recordings (3 seconds) of SLO-1 expressed in oocytes at $100 \mathrm{mV}$. Fifty-millimolar ethanol potentiated the probability of opening in control recordings, which was blocked in the presence of $500 \mathrm{nM}$ LS10. (E) The $\mathrm{P}_{\mathrm{o}}$ was measured each minute using two (3-second) traces plotted relative to baseline as mean \pm S.E.M. (F) Largest potentiation in $\mathrm{P}_{\mathrm{o}}$ after ethanol application is plotted relative to baseline for control and LS10-preincubated patches. The peak $\mathrm{P}_{\mathrm{o}}$ measured over a 1.5-minute interval of approximately three (3-second) traces was compared with baseline for five or more (3-second) traces via paired Student's $t$ tests, $* P<0.05, N=4$ to 5 .
(Pietrzykowski et al., 2008). Future uses of this platform could strive to select peptides that differentially restrict ethanol action dependent upon channel composition or post-translational modifications. Phage display alone has had moderate success in selecting peptides that show composition-dependent modulation of glycine receptor function (Cornelison et al., 2016). Furthermore, worms used in our secondary behavioral screen could be humanized to express different $\mathrm{BK} \alpha$ isoforms or phosphomutants. Combined $\mathrm{BK} \alpha$ and auxiliary subunit expression may even be possible; $C$. elegans does not express mammalian-like auxiliary subunits that would require null backgrounds, although it has not yet been shown whether BK channel auxiliary subunits traffic to the plasma membrane in worm. LS10 serves as proof-of-concept that this screening platform could provide novel tools to further our understanding of how alcohol acts at the BK channel to exert influence over CNS function.

\section{Acknowledgments}

Some strains were provided by the Caenorhabditis Genetics Center, which is funded by the National Institutes of Health Office of Research Infrastructure Programs (P40 OD010440).

\section{Authorship Contributions}

Participated in research design: Scott, Mihic, Aldrich, Pierce.
Conducted experiments: Scott, Iyer, Philpo, Avalos, Wu, Shi, Prakash, Nguyen.

Performed data analysis: Scott, Iyer, Philpo, Avalos, Wu, Shi, Prakash, Nguyen.

Wrote or contributed to the writing of the manuscript: Scott, Mihic, Aldrich, Pierce.

\section{References}

Alqadah A, Hsieh YW, Schumacher JA, Wang X, Merrill SA, Millington G, Bayne B, Jorgensen EM, and Chuang CF (2016) SLO BK potassium channels couple gap junctions to inhibition of calcium signaling in olfactory neuron diversification. PLoS Genet 12:e1005654.

Andersson L, Blomberg L, Flegel M, Lepsa L, Nilsson B, and Verlander M (2000) Large-scale synthesis of peptides. Biopolymers 55:227-250.

Bettinger JC and Davies AG (2014) The role of the BK channel in ethanol response behaviors: evidence from model organism and human studies. Front Physiol 5:346. Blednov YA, Benavidez JM, Homanics GE, and Harris RA (2012) Behavioral characterization of knockin mice with mutations M287L and Q266I in the glycine receptor $\alpha 1$ subunit. J Pharmacol Exp Ther 340:317-329.

Borghese CM, Blednov YA, Quan Y, Iyer SV, Xiong W, Mihic SJ, Zhang L, Lovinger DM, Trudell JR, Homanics GE, et al. (2012) Characterization of two mutations, M287L and Q266I, in the $\alpha 1$ glycine receptor subunit that modify sensitivity to alcohols. J Pharmacol Exp Ther 340:304-316.

Brenner S (1974) The genetics of Caenorhabditis elegans. Genetics 77:71-94.

Brodie MS and Appel SB (2000) Dopaminergic neurons in the ventral tegmental area of $\mathrm{C} 57 \mathrm{BL} / 6 \mathrm{~J}$ and DBA/2J mice differ in sensitivity to ethanol excitation. Alcohol Clin Exp Res 24:1120-1124.

Brodie MS, Scholz A, Weiger TM, and Dopico AM (2007) Ethanol interactions with calcium-dependent potassium channels. Alcohol Clin Exp Res 31:1625-1632.

Bukiya AN, Kuntamallappanavar G, Edwards J, Singh AK, Shivakumar B and Dopico AM (2014) An alcohol-sensing site in the calcium- and voltage-gated, large conductance potassium (BK) channel. Proc Natl Acad Sci USA 111: 9313-9318. 
Bull K, Cook A, Hopper NA, Harder A, Holden-Dye L, and Walker RJ (2007) Effects of the novel anthelmintic emodepside on the locomotion, egg-laying behaviour and development of Caenorhabditis elegans. Int J Parasitol 37:627-636.

Chen L, Tian L, MacDonald SHF, McClafferty H, Hammond MSL, Huibant JM, Ruth P, Knaus HG, and Shipston MJ (2005) Functionally diverse complement of large conductance calcium- and voltage-activated potassium channel (BK) $\alpha$-subunits generated from a single site of splicing. J Biol Chem 280:33599-33609.

Chu B and Treistman SN (1997) Modulation of two cloned potassium channels by 1-alkanols demonstrates different cutoffs. Alcohol Clin Exp Res 21:1103-1107.

C. elegans Sequencing Consortium (1998) Genome sequence of the nematode C. elegans: a platform for investigating biology. Science 282:2012-2018.

Cornelison GL, Pflanz NC, Tipps ME, and Mihic SJ (2016) Identification and characterization of heptapeptide modulators of the glycine receptor. Eur J Pharmacol 780:252-259.

Davies AG, Pierce-Shimomura JT, Kim H, VanHoven MK, Thiele TR, Bonci A, Bargmann CI, and McIntire SL (2003) A central role of the BK potassium channel in behavioral responses to ethanol in C. elegans. Cell 115:655-666.

Davis SJ, Scott LL, Hu K, and Pierce-Shimomura JT (2014) Conserved single residue in the BK potassium channel required for activation by alcohol and intoxication in C. elegans. J Neurosci 34:9562-9573.

Dopico AM (2003) Ethanol sensitivity of $\mathrm{BK}_{\mathrm{Ca}}$ channels from arterial smooth muscle does not require the presence of the $\beta_{1}$-subunit. Am J Physiol Cell Physiol 284:C1468-C1480.

Dopico AM, Anantharam V, and Treistman SN (1998) Ethanol increases the activity of $\mathrm{Ca}^{++}$-dependent $\mathrm{K}^{+}$(mslo) channels: functional interaction with cytosolic $\mathrm{Ca}^{++}$ $J$ Pharmacol Exp Ther 284:258-268.

Dopico AM, Bukiya AN, Kuntamallappanavar G, and Liu J (2016) Modulation of BK channels by ethanol. Int Rev Neurobiol 128:239-279.

Dopico AM, Bukiya AN, and Martin GE (2014) Ethanol modulation of mammalian BK channels in excitable tissues: molecular targets and their possible contribution to alcohol-induced altered behavior. Front Physiol 5:466.

Du W, Bautista JF, Yang H, Diez-Sampedro A, You SA, Wang L, Kotagal P, Lüders HO, Shi J, Cui J, et al. (2005) Calcium-sensitive potassium channelopathy in human epilepsy and paroxysmal movement disorder. Nat Genet 37:733-738.

Ghezzi A and Atkinson NS (2011) Homeostatic control of neural activity: a Drosophila model for drug tolerance and dependence, in International Review of Neurobiology (Nigel A ed) pp 23-50, Academic Press, Cambridge, MA

Glauser DA, Johnson BE, Aldrich RW, and Goodman MB (2011) Intragenic alternative splicing coordination is essential for Caenorhabditis elegans slo-1 gene function. Proc Natl Acad Sci USA 108:20790-20795.

Gudmundsson OS, Pauletti GM, Wang W, Shan D, Zhang H, Wang B, and Borchardt RT (1999) Coumarinic acid-based cyclic prodrugs of opioid peptides that exhibit metabolic stability to peptidases and excellent cellular permeability. Pharm Res 16:7-15.

Hoshi T, Pantazis A, and Olcese R (2013) Transduction of voltage and $\mathrm{Ca}^{2+}$ signals by Slo1 BK channels. Physiology (Bethesda) 28:172-189.

Howard RJ, Trudell JR, and Harris RA (2014) Seeking structural specificity: direct modulation of pentameric ligand-gated ion channels by alcohols and general anesthetics. Pharmacol Rev 66:396-412.

Jakab M, Weiger TM, and Hermann A (1997) Ethanol activates maxi $\mathrm{Ca}^{2+}$-activated $\mathrm{K}^{+}$channels of clonal pituitary (GH3) cells. J Membr Biol 157:237-245.

Johnson BE, Glauser DA, Dan-Glauser ES, Halling DB, Aldrich RW, and Goodman MB (2011) Alternatively spliced domains interact to regulate BK potassium channel gating. Proc Natl Acad Sci USA 108:20784-20789.

Kreifeldt M, Le D, Treistman SN, Koob GF, and Contet C (2013) BK channel $\beta 1$ and $\beta 4$ auxiliary subunits exert opposite influences on escalated ethanol drinking in dependent mice. Front Integr Nuerosci 7:105.

Kwok TC, Ricker N, Fraser R, Chan AW, Burns A, Stanley EF, McCourt P, Cutler SR, and Roy PJ (2006) A small-molecule screen in C. elegans yields a new calcium channel antagonist. Nature 441:91-95.

Lai CH, Chou CY, Ch'ang LY, Liu CS, and Lin W (2000) Identification of novel human genes evolutionarily conserved in Caenorhabditis elegans by comparative proteomics. Genome Res 10:703-713.

Liu L, Hendrickson LM, Guildford MJ, Zhao-Shea R, Gardner PD, and Tapper AR (2013) Nicotinic acetylcholine receptors containing the $\alpha 4$ subunit modulate alcohol reward. Biol Psychiatry 73:738-746.

Martin GE, Hendrickson LM, Penta KL, Friesen RM, Pietrzykowski AZ, Tapper AR, and Treistman SN (2008) Identification of a BK channel auxiliary protein con trolling molecular and behavioral tolerance to alcohol. Proc Natl Acad Sci USA 105:17543-17548.

Meredith AL, Thorneloe KS, Werner ME, Nelson MT, and Aldrich RW (2004) Overactive bladder and incontinence in the absence of the BK large conductance $\mathrm{Ca}^{2+}$-activated $\mathrm{K}^{+}$channel. J Biol Chem 279:36746-36752.
Mondal S, Hegarty E, Sahn JJ, Scott LL, Gökçe SK, Martin C, Ghorashian N, Satarasinghe PN, Iyer S, Sae-Lee W, et al. (2018) High-content microfluidic screening platform used to identify $\sigma 2 \mathrm{R} / \mathrm{Tmem} 97$ binding ligands that reduce agedependent neurodegeneration in C. elegans SC_APP model. ACS Chem Neurosci 9 : 1014-1026.

Mulholland PJ, Hopf FW, Bukiya AN, Martin GE, Liu J, Dopico AM, Bonci A Treistman SN, and Chandler LJ (2009) Sizing up ethanol-induced plasticity: the role of small and large conductance calcium-activated potassium channels. Alcohol Clin Exp Res 33:1125-1135.

National Research Council (2011) Guide for the Care and Use of Laboratory Animals: Eighth Edition. Washington, DC: The National Academies Press. https://doi.org/ $10.17226 / 12910$

Pietrzykowski AZ, Friesen RM, Martin GE, Puig SI, Nowak CL, Wynne PM, Siegelmann HT, and Treistman SN (2008) Post-transcriptional regulation of BK channel splice variant stability by miR-9 underlies neuroadaptation to alcohol. Neuron 59:274-287.

Röttgen P and Collins J (1995) A human pancreatic secretory trypsin inhibitor presenting a hypervariable highly constrained epitope via monovalent phagemid display. Gene 164:243-250.

Rüttiger L, Sausbier M, Zimmermann U, Winter H, Braig C, Engel J, Knirsch M, Arntz C, Langer P, Hirt B, et al. (2004) Deletion of the $\mathrm{Ca}^{2+}$-activated potassium (BK) $\alpha$-subunit but not the BK $\beta 1$-subunit leads to progressive hearing loss. Proc Natl Acad Sci USA 101:12922-12927.

Scott LL, Brecht EJ, Philpo A, Iyer S, Wu NS, Mihic SJ, Aldrich RW, Pierce J, and Walton JP (2017a) A novel BK channel-targeted peptide suppresses sound evoked activity in the mouse inferior colliculus. Sci Rep 7:42433.

Scott LL, Davis SJ, Yen RC, Ordemann GJ, Nordquist SK, Bannai D, and Pierce JT (2017b) Behavioral deficits following withdrawal from chronic ethanol are influenced by SLO channel function in Caenorhabditis elegans. Genetics 206: $1445-1458$.

Sonnhammer EL and Durbin R (1997) Analysis of protein domain families in Caenorhabditis elegans. Genomics 46:200-216.

Tipps ME, Lawshe JE, Ellington AD, and Mihic SJ (2010) Identification of nove specific allosteric modulators of the glycine receptor using phage display. J Biol Chem 285:22840-22845.

Treistman SN and Martin GE (2009) BK channels: mediators and models for alcohol tolerance. Trends Neurosci 32:629-637.

Wallner M, Meera P, and Toro L (1999) Molecular basis of fast inactivation in voltage and $\mathrm{Ca}^{2+}$-activated $\mathrm{K}^{+}$channels: a transmembrane $\beta$-subunit homolog. Proc Natl Acad Sci USA 96:4137-4142.

Walters FS, Covarrubias M, and Ellingson JS (2000) Potent inhibition of the aortic smooth muscle maxi-K channel by clinical doses of ethanol. Am J Physiol Cell Physiol 279:C1107-C1115.

Wang ZW, Saifee O, Nonet ML, and Salkoff L (2001) SLO-1 potassium channels control quantal content of neurotransmitter release at the $C$. elegans neuromuscular junction. Neuron 32:867-881.

White RE, Schonbrunn A, and Armstrong DL (1991) Somatostatin stimulates $\mathrm{Ca}^{2+}$-activated $\mathrm{K}^{+}$channels through protein dephosphorylation. Nature 351: $570-573$.

Wilkemeyer MF, Chen SY, Menkari CE, Brenneman DE, Sulik KK, and Charness ME (2003) Differential effects of ethanol antagonism and neuroprotection in peptide fragment NAPVSIPQ prevention of ethanol-induced developmental toxicity. Proc Natl Acad Sci USA 100:8543-8548.

Xia XM, Ding JP, and Lingle CJ (2003) Inactivation of BK channels by the $\mathrm{NH}_{2}$ terminus of the $\beta 2$ auxiliary subunit: an essential role of a terminal peptide segment of three hydrophobic residues. J Gen Physiol 121:125-148.

Yu M, Liu SL, Sun PB, Pan H, Tian CL, and Zhang LH (2016) Peptide toxins and small-molecule blockers of BK channels. Acta Pharmacol Sin 37:56-66.

Zhang Y, Shi ZG, Wang ZH, Li JG, Chen JY, and Zhang C (2014) Effects of amyloid $\beta$-peptide fragment $31-35$ on the $\mathrm{BK}$ channel-mediated $\mathrm{K}^{+}$current and intracellular free $\mathrm{Ca}^{2+}$ concentration of hippocampal CA1 neurons. Neurosci Lett 568:72-76.

Address correspondence to: Dr. Jonathan T. Pierce, Department of Neuroscience, The University of Texas at Austin, 2506 Speedway, Mail Code C7350, Austin, TX 78712. E-mail: jonps@austin.utexas.edu; or Dr. Luisa L. Scott, Department of Neuroscience, The University of Texas at Austin, 2506 Speedway, Mail Code C7350, Austin, TX 78712. E-mail: luisa_scott@ utexas.edu 